\title{
Electronic structure and exchange interactions in the manganese-based pyrochlore oxides
}

\author{
S. K. Mishra* and S. Satpathy \\ Department of Physics and Astronomy, University of Missouri, Columbia, Missouri 65211
}

(Received 7 October 1997; revised manuscript received 16 January 1998)

\begin{abstract}
We describe the ferromagnetism in the manganese pyrochlores, such as $\mathrm{Tl}_{2} \mathrm{Mn}_{2} \mathrm{O}_{7}$ and $\mathrm{Sc}_{2} \mathrm{Mn}_{2} \mathrm{O}_{7}$, in terms of the interplay between superexchange, Zener double exchange, and indirect exchange, the first being antiferromagnetic (AF) while the last two are ferromagnetic. The tendency towards the antiferroalignment of the localized Mn spins is significantly weakened due to (i) the presence of spin frustration in the Mn sublattice and (ii) the large bend in the Mn-O-Mn bond, which reduces the magnitude of the AF superexchange. This helps the ferromagnetic interaction terms, viz., the indirect exchange and the Zener double exchange, to dominate. The indirect exchange on the Mn-O-Mn bond, which is modeled by a coupling of the $\mathrm{O} 2 p$ electrons to the conduction-band states, produces a weak ferromagnetism in the insulating pyrochlores. In the metallic pyrochlores, the indirect exchange is also present, but it is now supplemented by the Zener double exchange, making the ferromagnetism more robust, as indicated from a higher value of the Curie temperature $T_{c}$. Density-functional calculations for the metallic $\mathrm{Tl}_{2} \mathrm{Mn}_{2} \mathrm{O}_{7}$ pyrochlore, show the itinerant Zener carriers mediating double-exchange to be electrons in the $\Gamma_{1}$ minority spin band crossing the Fermi energy, with predominantly $\mathrm{Mn} 3 d$, Tl $6 s$, and $\mathrm{O} 2 p$ characters. These Zener carriers move in a lattice of localized Mn $t_{2 g}$ spins, with the carrier spins aligned antiparallel to the localized spins. [S0163-1829(98)05335-1]
\end{abstract}

\section{INTRODUCTION}

The manganese-based pyrochlore compounds, such as $\mathrm{Tl}_{2} \mathrm{Mn}_{2} \mathrm{O}_{7}$, are an intriguing class of manganese compounds. Unlike their perovskite counterparts, they are nominally not mixed valence, do not exhibit a strong Jahn-Teller effect, have presumably a weak electron-phonon coupling, and yet exhibit the colossal magnetoresistive (CMR) behavior. ${ }^{1,2}$ The discovery of CMR in the pyrochlores is therefore of great significance to the understanding of the mechanism for CMR.

We address in this paper the issue concerning the nature of electron states and magnetic exchange interactions in the pyrochlores. Our analysis is based on results of densityfunctional studies of the electronic structure and on results of simple models that we develop for the magnetic exchange. We propose that the relatively weak ferromagnetism in the insulating pyrochlores is produced by an indirect exchange, while the ferromagnetism is stronger in the metallic pyrochlores because of the additional ferromagnetic (FM) coupling due to double exchange.

The Zener double exchange $\mathrm{e}^{3-5}$ relies on the presence of two types of spins in the lattice, viz., the itinerant spins (Zener carriers) and a lattice of localized spins. Because a certain alignment of the itinerant spins with respect to the localized spins is required by the physics of the problem (e.g., Hund's rule favors a parallel spin alignment), the Zener carriers can move about freely if the localized spins are aligned ferromagnetically with respect to one another. If a sufficient number of carriers is present, typically $\approx 10 \%$ for the perovskite manganites, then they can turn an otherwise antiferromagnetic lattice ferromagnetic.

The double exchange (DE) was originally conceived by Zener to be produced by the simultaneous hopping of a Mn electron to an oxygen atom and an oxygen electron to the other $\mathrm{Mn}$ atom on a Mn-O-Mn bond, so that the oxygen shell remains full and in the process the valence of the two Mn atoms are interchanged. It has been suggested that the DE mechanism may be absent in the pyrochlores because of a large bend in the Mn-O-Mn bond angle and because of nominal lack of mixed valence of the Mn ions. In this paper, we shall argue that a double exchange mechanism is indeed operational in the metallic pyrochlores and the system, even though stoichiometric, may be considered to be "internally doped,' thereby producing the necessary Zener carriers. The competing antiferromagnetic (AF) superexchange between the localized spins is shown to be weakened by the large bend of the Mn-O-Mn bond, which helps the FM interaction terms to be dominant.

\section{ELECTRONIC BAND STRUCTURE OF $\mathrm{Tl}_{2} \mathrm{Mn}_{2} \mathrm{O}_{7}$}

To understand the nature of the relevant electron states, we have performed a local-spin-density approximation (LSDA) calculation of the electronic structure for a specific metallic pyrochlore compound, viz., $\mathrm{Tl}_{2} \mathrm{Mn}_{2} \mathrm{O}_{7}$, in the experimental $F d \overline{3} m$ structure, ${ }^{6,7}$ using the linear muffin-tin orbitals (LMTO) method. ${ }^{8}$ The electron band structures, shown in Fig. 1, agree with those presented earlier by Singh $^{9}$ using the linearized augmented plane-wave method. In addition to the bands, we have also shown the symmetries of the wave functions at the $\Gamma$ point in the Brillouin zone, which is useful in determining the orbital components of the electron states from the symmetry information presented in Table I.

The calculated spin magnetic moment of $2.97 \mu_{B}$ is consistent with the measured value of $2.74 \mu_{B}$ (Ref. 1) or $2.59 \mu_{B} .{ }^{6}$ The experimental values are probably underestimates in view of impurities present in the sample and due to lack of complete saturation of magnetization. ${ }^{1}$ The small deviation of the calculated spin moment from an integer value is consistent with the near-complete filling of both the majority and the minority spin bands. In fact, the number of 


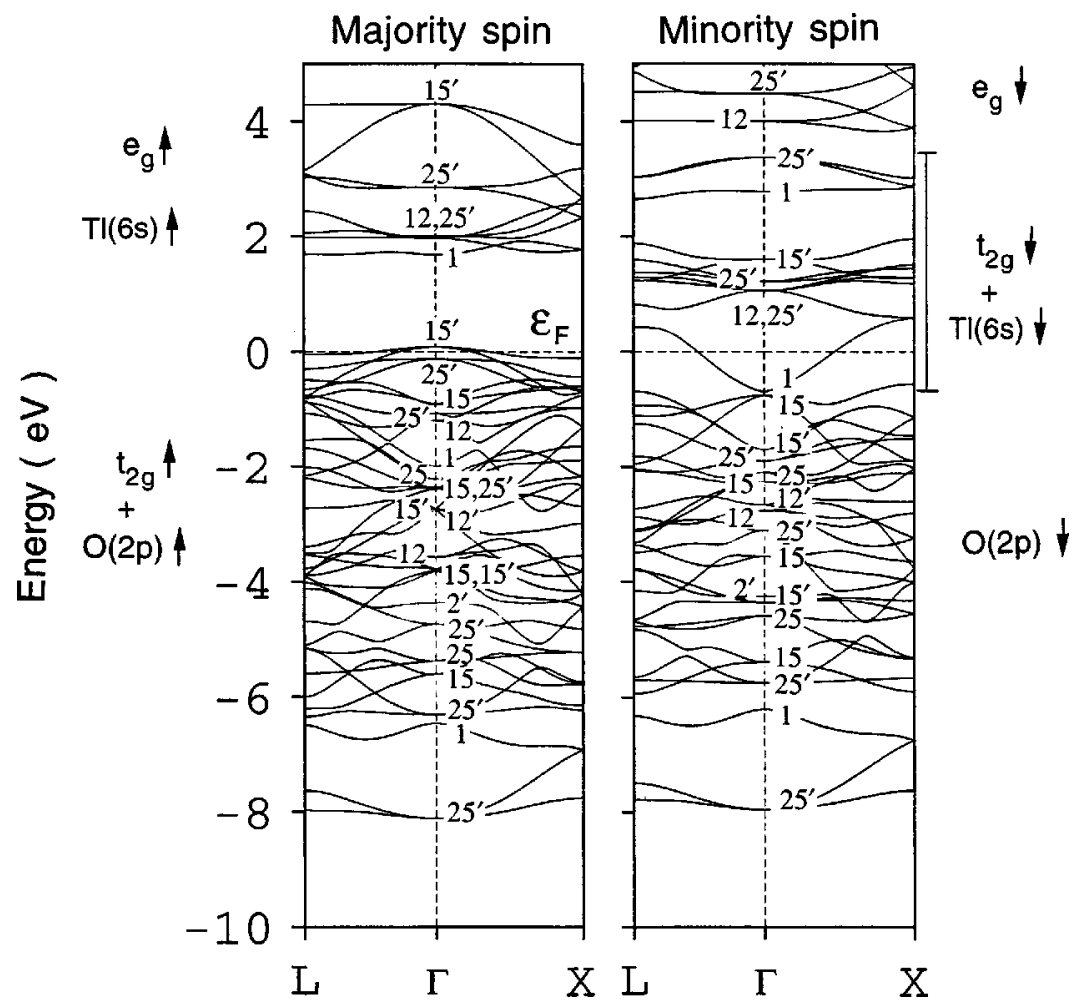

FIG. 1. Energy-band structure of ferromagnetic $\mathrm{Tl}_{2} \mathrm{Mn}_{2} \mathrm{O}_{7}$ calculated in the local-spin-density approximation.

electrons in the $\Gamma_{1}$ pocket at the Fermi energy may be computed from this deviation and it turns out to be about $0.03 e^{-}$ per formula unit.

The basic features of the band structures are summarized as follows. The occupied majority spin bands consist of a joint $\mathrm{O} 2 p+\mathrm{Mn} t_{2 g}$ band of $\sim 8 \mathrm{eV}$ width, with a small pocket of $\Gamma_{15}^{\prime}$ holes. An important feature of the band structure is a free-electron-like $\Gamma_{1}$ minority spin band crossing the Fermi energy. Since the $\Gamma_{15}^{\prime}$ holes have a large mass (small band dispersion), these states are expected to be unimportant as current carriers and may indeed be localized by the presence of a small amount of impurity. The electrons in the $\Gamma_{1}$ pocket, therefore, are the important mobile carriers and they are the equivalent of the $\mathrm{Mn} e_{g}$ Zener carriers in the perovskites. The picture of electronic structure that then emerges is shown in Fig. 2. The band results are consistent with the $\mathrm{x}$-ray-absorption spectroscopy data indicating the presence of doping into the $\mathrm{Tl} 6 s$-like band. ${ }^{10,11}$

To illustrate the nature of these $\Gamma_{1}$ carriers, we have projected out the partial density of states (DOS) (shown in the top panel of Fig. 3), from which we find that the wavefunction character of the $\Gamma_{1}$ pocket consists primarily of $\mathrm{Mn}$ $t_{2 g}$ and $\mathrm{Tl} 6 s$ characters, and some $\mathrm{O}(2) 2 p$ character. The two types of oxygen atoms $\mathrm{O}(1)$ and $\mathrm{O}(2)$ form, respectively, the $\mathrm{Tl}_{4} \mathrm{O}(1)$ tetrahedra and the $\mathrm{MnO}(2)_{6}$ octahedra. $^{7,12}$ It is interesting that the $\mathrm{Mn} e_{g}$ orbitals responsible for the Zener carriers in the perovskites contribute very little in the case of the pyrochlores. In fact, from symmetry considerations (see Table I), the Mn $e_{g}$ orbitals cannot contribute to the $\Gamma_{1}$ state at the center of the Brillouin zone. This means that the contribution of the $\mathrm{Mn} e_{g}$ orbitals to the $\Gamma_{1}$ pocket is greatly diminished, even though the symmetry argument holds strictly at the $\Gamma$ point only. Contribution from the $\mathrm{O}(1) 2 p$ orbitals is also small for the same reason.

TABLE I. Irreducible representations of the $\mathrm{O}_{h}$ group spanned by various orbitals in the pyrochlore structure $\mathrm{Tl}_{2} \mathrm{Mn}_{2} \mathrm{O}(1) \mathrm{O}(2)_{6} . N$ is the number of orbitals per unit cell. The oxygen occurring at the center of the $\mathrm{Tl}$ tetrahedra is denoted by $\mathrm{O}(1)$, while the $\mathrm{O}(2)$ atoms form the $\mathrm{MnO}_{6}$ octahedra.

\begin{tabular}{|c|c|c|c|c|c|c|c|c|c|c|c|}
\hline Orbitals & $N$ & $\Gamma_{1}$ & $\Gamma_{2}$ & $\Gamma_{12}$ & $\Gamma_{15}$ & $\Gamma_{25}$ & $\Gamma_{1}^{\prime}$ & $\Gamma_{2}^{\prime}$ & $\Gamma_{12}^{\prime}$ & $\Gamma_{15}^{\prime}$ & $\Gamma_{25}^{\prime}$ \\
\hline $\mathrm{Mn} / \mathrm{Tl}(s)$ & 4 & 1 & & & & & & & & & 1 \\
\hline $\mathrm{Mn} / \mathrm{Tl}(p)$ & 12 & & & & 2 & 1 & & 1 & 1 & & \\
\hline $\mathrm{Mn} / \mathrm{Tl}(d) t_{2 g}$ & 12 & 1 & & 1 & & & & & & 1 & 2 \\
\hline $\mathrm{Mn} / \mathrm{Tl}(d) e_{g}$ & 8 & & & 1 & & & & & & 1 & 1 \\
\hline $\mathrm{O}(1)(s)$ & 2 & 1 & & & & & & 1 & & & \\
\hline $\mathrm{O}(1)(p)$ & 6 & & & & 1 & & & & & & 1 \\
\hline $\mathrm{O}(2)(s)$ & 12 & 1 & & 1 & 1 & & & 1 & 1 & & 1 \\
\hline $\mathrm{O}(2)(p)$ & 36 & 1 & & 1 & 3 & 2 & & 1 & 1 & 2 & 3 \\
\hline
\end{tabular}




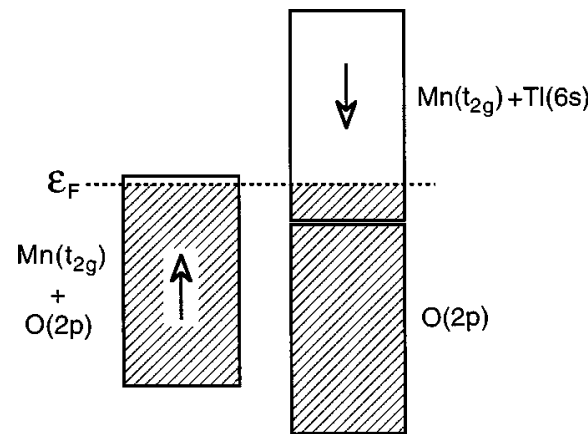

FIG. 2. Schematic band structure corresponding to Fig. 1.

The nature of the $\Gamma_{1}$ electrons is further illustrated from the charge-density contour plots (Fig. 4), which were obtained by summing over the electron charge density of the occupied states just below $E_{f}$ in this pocket. The contributions from the various atoms are: $\mathrm{Tl}_{2}=2 \times 12 \%, \mathrm{Mn}_{2}=2$ $\times 18 \%, \mathrm{O}(1)_{1}=1 \times 7 \%, \mathrm{O}(2)_{6}=6 \times 3 \%$, and the remaining $15 \%$ coming from the empty sphere orbitals in the LMTO method.

It may be remarked here that the Ruderman-KittelKasuya-Yosida (RKKY) interaction, ${ }^{13}$ describing the interaction between two localized spins mediated via conduction electrons (such as the $\Gamma_{1}$ electrons in $\mathrm{Tl}_{2} \mathrm{Mn}_{2} \mathrm{O}_{7}$ here), is obtained by assuming a small perturbation of the conduction electrons by a local spin. The perturbation produces halos in
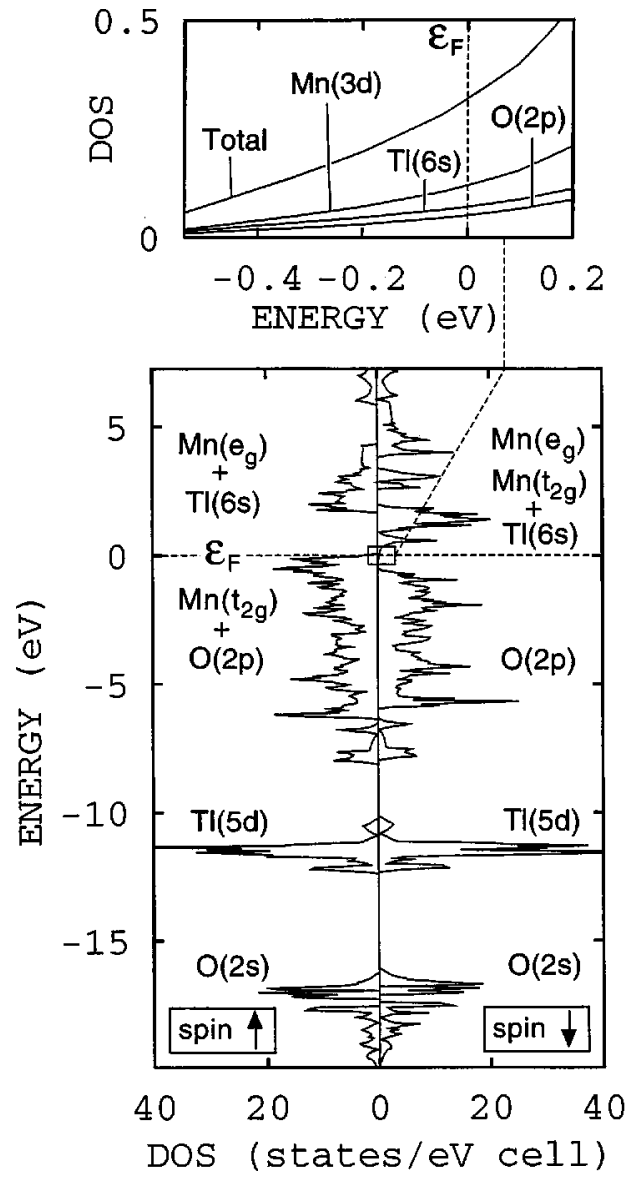

FIG. 3. One-electron density of states for $\mathrm{Tl}_{2} \mathrm{Mn}_{2} \mathrm{O}_{7}$. The top panel shows the partial DOS of the minority electrons, corresponding to the $\Gamma_{1}$ band near the Fermi energy.

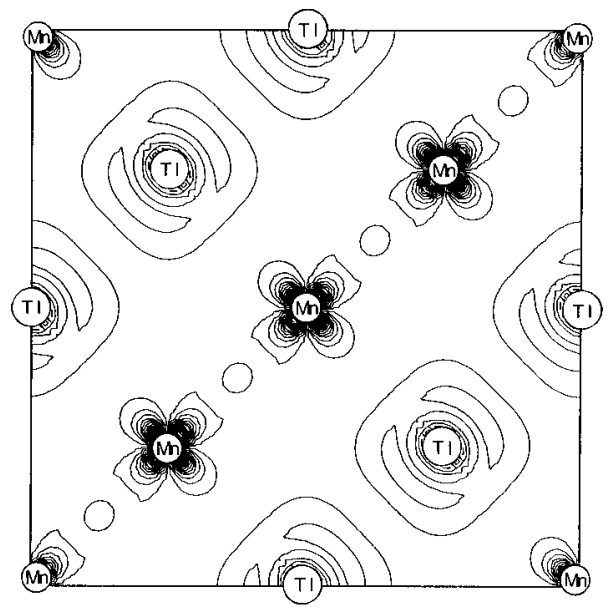

FIG. 4. Electronic charge-density contours of the minority spin $\Gamma_{1}$ band on a plane containing $\mathrm{Mn}$ and $\mathrm{Tl}$ atoms. The charge density is spread out in the entire solid, consistent with the itinerant character of this band.

the spin density, i.e., alternating excesses of spin-up and spin-down densities. A second spin aligns either ferro- or antiferromagnetically with the first spin depending on the predominant spin density at its site.

The RKKY ideas work very well for dilute magnetic systems. In the pyrochlores, on the other hand, the entire lattice of Mn $t_{2 g}$ spins affect the conduction electrons perturbing them strongly. The perturbation is so strong in fact that the free-electron-like $\Gamma_{1}$ band is completely spin polarized as seen from the band structure. This being the case, only one type of conduction spins is present, which leads to a FM interaction between the Mn atoms at all distances. This is in contrast to the oscillating RKKY interaction originating from the oscillating spin-up and -down densities. The magnetic interaction carried by such strong perturbation of the conduction spins is best described in terms of the standard Zener double-exchange model.

\section{DOUBLE-EXCHANGE IN METALLIC PYROCHLORES}

In this section, we describe the double-exchange mechanism for $\mathrm{Tl}_{2} \mathrm{Mn}_{2} \mathrm{O}_{7}$, which may be relevant for the entire class of metallic pyrochlores, assuming that their band structures are similar.

The mechanism involving the $\Gamma_{1}$ carriers and the Mn core spins is described by the standard DE Hamiltonian:

$$
H=-\sum_{\langle i j\rangle} J \vec{S}_{i} \cdot \vec{S}_{j}-t \sum_{\langle i j\rangle, \sigma} c_{i \sigma}^{\dagger} c_{j \sigma}+\text { H.c. }+\sum_{i} J_{H} \vec{S}_{i} \cdot \vec{s}_{i},
$$

where $\vec{S}(\vec{s})$ denotes the spins of the localized (itinerant) electrons.

There are, however, a few differences from the DE in the perovskite manganites. First, the majority-spin $t_{2 g}$ electrons forming the core spins are much less localized in the pyrochlores, and may have to be treated more accurately. To a first approximation, however, they still may be considered classical and localized with $S=3 / 2$. Second, there is an important difference in the nature of the Zener carriers. Our band calculations show that these consist of the $\Gamma_{1}$ electron pocket with their wave functions made up of $\mathrm{Mn} t_{2 g} \downarrow, \mathrm{Tl}$ $6 s \downarrow$, and O $2 p \downarrow$ orbitals. The Mn $t_{2 g} \downarrow$ part of the wave 
function may be expected to interact via $\mathrm{DE}$ with the localized Mn $t_{2 g} \uparrow$ spins. What gives these Mn $t_{2 g} \downarrow$ electrons a delocalized character is the admixture with the free-electronlike $\mathrm{Tl} 6 s$ and $\mathrm{O}(2) 2 p$ states. Being of itinerant character, these electrons are expected to both conduct well and mediate double exchange. Notice that the Coulomb interaction terms in the Hamiltonian that led to the very interesting charge-ordering phenomena in the perovskites ${ }^{14,15}$ are unimportant for the pyrochlores because of the small number of Zener carriers and they have been omitted from the Hamiltonian, Eq. (1).

The nature of the Zener carriers is in direct contrast with the $e_{g} \uparrow$ electrons in the perovskites, ${ }^{16}$ where the strong Hund's rule energy cost $J_{H}$ ensured that the spin of the $e_{g}$ electrons line up with the $t_{2 g}$ spins on Mn. The parallel alignment of the itinerant and the localized spins is absent in the pyrochlores and it is replaced by the opposite tendency, viz., that the $\Gamma_{1}$ conduction electrons now prefer to be aligned antiparallel to the localized $\mathrm{Mn} t_{2 g}$ spins. Thus $J_{H}$ is in effect negative. This is not surprising and is the result of a combination of (i) the relatively small $\mathrm{Mn} 3 d$ component of the $\Gamma_{1}$ electrons and (ii) the band-structure hybridization effects.

Based on the exchange splitting between the $\Gamma_{1}$ spin-up and -down bands of $\sim 2 \mathrm{eV}$, the magnitude of the effective $J_{H}$ is slightly smaller than the same quantity in the manganites. As already emphasized by Zener ${ }^{3}$ and by Anderson and Hasegawa, ${ }^{4}$ the DE interaction is not altered by whether the conduction spins prefer to align parallel or antiparallel with the localized spins and the pyrochlores are indeed an example of the latter case.

The total number of electrons occupying the $\Gamma_{1} \downarrow$ band as obtained from our calculation is about $0.03 e^{-}$per formula unit as compared to the nominal carrier concentration of $0.001-0.005 e^{-}$suggested from Hall measurements. ${ }^{1}$ However, the experimental estimate suffers from two deficiencies: (i) the standard expression for the Hall coefficient in terms of the electron number assumes a spherical Fermi surface and (ii) there may be charge compensation coming from the impurities in the experimental pyrochlore samples. In addition to these, the presence of both an electron and a hole pocket complicates the situation. All these would render the measured value of the electron number inaccurate at best. Nevertheless, both experiments and the band calculations indicate that the number of carriers is undoubtedly small but nonzero.

The question now is whether the ferromagnetic DE produced by such a small number of Zener carriers is sufficient to overcome the AF superexchange interaction among the core spins. A simple estimate following De Gennes' argument ${ }^{5}$ would indicate a $\mathrm{DE}$ interaction $J_{\mathrm{DE}} \sim(W / 2)$ $\times x$, where $W$ is the bandwidth $(W \sim 2 \mathrm{eV})$ and $x$ is the carrier concentration $(x \sim 0.015 \bar{e} / \mathrm{Mn}$ atom), resulting in a FM $J_{\mathrm{DE}} \sim 15 \mathrm{meV}$ for $\mathrm{Tl}_{2} \mathrm{Mn}_{2} \mathrm{O}_{7}$, which is indeed sizable. In addition, the ferromagnetic $\mathrm{DE}$ interaction is very effective in the pyrochlores, because of the fact that the AF superexchange is considerably weakened due to two factors. First, there is a considerable deviation from the linear $\mathrm{Mn}-\mathrm{O}-\mathrm{Mn}$ bond with a Mn-O-Mn bend angle of $\approx 133^{\circ} .{ }^{12}$ This weakens the AF superexchange between the Mn core spins mediated via the $\mathrm{O}(2 p)$ electrons as we shall see below. Second,

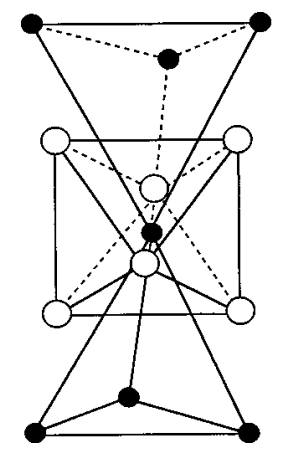

FIG. 5. Connectivity of the Mn sublattice indicating lattice frustration and also showing the octahedral arrangement of the oxygen atoms in the pyrochlore structure. Black (white) circles denote Mn (O) atoms.

the Mn sublattice being magnetically frustrated (see Fig. 5), a net antiferromagnetic interaction cannot be taken full advantage of. The topology of the Mn sublattice is in fact similar to that of the spinel $B$ sublattice, e.g., of magnetite $\left(\mathrm{Fe}_{3} \mathrm{O}_{4}\right)$, where the lattice frustration causes the well-known charge order-disorder transition (Verwey transition). ${ }^{17,18}$ The weakening of the AF interaction is sufficient that the indirect exchange (discussed in Sec. V) is able to tip the energy balance in favor of a ferromagnetic system, which is further strengthened by the ferromagnetic DE interaction in the metallic pyrochlores.

\section{EFFECT OF THE Mn-O-Mn BOND ANGLE ON SUPEREXCHANGE}

To illustrate the reduction of the superexchange interaction with the Mn-O-Mn bond angle, we consider the origin of the AF exchange interaction within a simple model. The spirit of the model is similar to the recent works on the superexchange interaction in the perovskite manganites. ${ }^{19,20}$ That the bend in the Mn-O-Mn bond reduces the antiferromagnetic superexchange has been, in fact, known for a long time, ${ }^{21}$ what we present below is an evaluation of the magnitude of this effect using a simple, exactly solvable model.

We consider a triatomic Mn-O-Mn system with a core $\mathrm{Mn}$ $t_{2 g}$ spin and a single "valence" orbital on each $\mathrm{Mn}$ atom onto which an $\mathrm{O} p$ electron can hop, provided that its spin is parallel to the Mn core spin. Thus, in effect, the Hund's rule energy cost is taken to be $\infty$, so that the antiparallel alignment of spins on the Mn atom is not allowed. Because of this and because we consider a single $\mathrm{Mn}$ valence orbital, a Coulomb interaction term on the $\mathrm{Mn}$ atom produces no effect in our model.

The superexchange interaction is mediated by the six oxygen $p$ electrons moving back and forth between the three atoms. However, since the out-of-plane oxygen $p_{z}$ orbital is not coupled by symmetry to the Mn orbital, only four of the six electrons participate in the superexchange interaction. We denote the Mn-O hopping matrix element by $t$ and ignore the Coulomb $U$ on the oxygen site. Inclusion of neither the oxygen $U$ nor a higher number of orbitals on the $\mathrm{Mn}$ atom presents any extra complications. However, it does not change the essential physics being considered here, so that our simple model suffices. ${ }^{22}$

When the core spins on the two Mn atoms are ferromag- 
netically aligned, there are four spin-up and two spin-down orbitals to be occupied by the four active $\mathrm{O} p$ electrons present in the problem. Since the on-site energy of the $\mathrm{O} p$ orbital is typically several $\mathrm{eV}$ below that of the "valence", orbital on the Mn atom, the ground state will have two spin-up and two spin-down electrons, moving in a sixdimensional $\left({ }^{4} C_{2} \times{ }^{2} C_{2}\right)$ configuration space. The Hamiltonian is given by

$$
H_{\uparrow \uparrow}=\left(\begin{array}{cccccc}
\epsilon_{d} & 0 & -t^{\prime \prime} & -t^{\prime} & 0 & 0 \\
0 & \epsilon_{d} & t^{\prime} & t^{\prime \prime} & 0 & 0 \\
-t^{\prime \prime} & t^{\prime} & 2 \epsilon_{d} & 0 & t^{\prime \prime} & t^{\prime} \\
-t^{\prime} & t^{\prime \prime} & 0 & 0 & t^{\prime} & t^{\prime \prime} \\
0 & 0 & t^{\prime \prime} & t^{\prime} & \epsilon_{d} & 0 \\
0 & 0 & t^{\prime} & t^{\prime \prime} & 0 & \epsilon_{d}
\end{array}\right),
$$

where $\epsilon_{d}$ is the on-site energy of the Mn orbital with respect to the $\mathrm{O} p$ orbital energy (zero of energy), $t^{\prime} \equiv t \cos (\theta / 2)$, and $t^{\prime \prime} \equiv t \sin (\theta / 2)$. The angle dependence here comes merely from the specific orientations of the oxygen $p_{x}$ and $p_{y}$ orbitals that affect the tight-binding matrix elements ${ }^{23}$ and it is by no means to be confused with the Anderson-Hasegawa hopping coming from double exchange. The basis set is in the order $\left|s_{1} x\right\rangle,\left|s_{1} y\right\rangle,\left|s_{1} s_{2}\right\rangle,|x y\rangle,\left|x s_{2}\right\rangle$, and $\left|y s_{2}\right\rangle$, referring to the two occupied spin-up orbitals. The two spin-down electrons can have just one possible configuration, viz., $|x y\rangle$, because, $J_{H}$ being $\infty$, the spin-down oxygen electrons are not allowed to hop to the Mn atoms. Here $x$ and $y$ denote the two in-plane oxygen $p$ orbitals and $s_{1}$ and $s_{2}$ denote the singly degenerate, $\Gamma_{1}$-like valence orbitals on the two Mn atoms with energy $\epsilon_{d}$.

The Hamiltonian (2) can be analytically diagonalized with the ground-state energy given by

$E_{\uparrow \uparrow}=\epsilon_{d}-\frac{1}{\sqrt{2}}\left[\epsilon_{d}^{2}+4 t^{2}+\sqrt{\epsilon_{d}^{4}+8 t^{2} \epsilon_{d}^{2}+16 t^{4} \sin ^{2} \theta}\right]^{1 / 2}$.

Now, in the case where the core spins of the two Mn atoms are aligned antiferromagnetically, there are three spin-up and an equal number of spin-down orbitals available to the four electrons, giving rise to a total of ${ }^{3} C_{2} \times{ }^{3} C_{2}=9$ possible configurations, viz., $\left|s_{1} x, s_{2} x\right\rangle,\left|s_{1} x, s_{2} y\right\rangle,\left|s_{1} x, x y\right\rangle$, $\left|s_{1} y, s_{2} x\right\rangle,\left|s_{1} y, s_{2} y\right\rangle,\left|s_{1} y, x y\right\rangle,\left|x y, s_{2} x\right\rangle,\left|x y, s_{2} y\right\rangle$, and $|x y, x y\rangle$, where the two orbitals on the left (right) side of the comma are occupied by spin-up (down) electrons. The Hamiltonian is then

$$
H_{\uparrow \downarrow}=\left(\begin{array}{ccccccccc}
2 \epsilon_{d} & 0 & -t^{\prime} & 0 & 0 & 0 & -t^{\prime} & 0 & 0 \\
0 & 2 \epsilon_{d} & -t^{\prime \prime} & 0 & 0 & 0 & 0 & -t^{\prime} & 0 \\
-t^{\prime} & -t^{\prime \prime} & \epsilon_{d} & 0 & 0 & 0 & 0 & 0 & -t^{\prime} \\
0 & 0 & 0 & 2 \epsilon_{d} & 0 & -t^{\prime} & t^{\prime \prime} & 0 & 0 \\
0 & 0 & 0 & 0 & 2 \epsilon_{d} & -t^{\prime \prime} & 0 & t^{\prime \prime} & 0 \\
0 & 0 & 0 & -t^{\prime} & -t^{\prime \prime} & \epsilon_{d} & 0 & 0 & t^{\prime \prime} \\
-t^{\prime} & 0 & 0 & t^{\prime \prime} & 0 & 0 & \epsilon_{d} & 0 & -t^{\prime} \\
0 & -t^{\prime} & 0 & 0 & t^{\prime \prime} & 0 & 0 & \epsilon_{d} & -t^{\prime \prime} \\
0 & 0 & -t^{\prime} & 0 & 0 & t^{\prime \prime} & -t^{\prime} & -t^{\prime \prime} & 0
\end{array}\right),
$$

with the ground-state energy given by

$$
E_{\uparrow \downarrow}=\epsilon_{d}-\sqrt{\epsilon_{d}^{2}+4 t^{2}}
$$

The exchange energy is given by the difference of the two ground-state energies:

$$
J_{e x}=E_{\uparrow \uparrow}-E_{\uparrow \downarrow}
$$

Note that $J_{e x}$ is analogous to $J S^{2}$ in Eq. (1). In the limit that $t / \epsilon_{d} \ll 1$, the exchange energy calculated from Eqs. (3), (5), and (6) reduces to the value

$$
J_{e x}=\frac{2 t^{4}}{\epsilon_{d}^{3}} \cos ^{2} \theta
$$

which is antiferromagnetic and this result may also be obtained by using the fourth-order perturbation theory. The $\cos ^{2} \theta$ dependence of the superexchange interaction has been well established from the variation of the Néel temperature in the antiferromagnetic $A \mathrm{FeO}_{3}$ compounds ( $\theta$ is the $\mathrm{Fe}$ O-Fe bond angle). ${ }^{24}$

The calculated angle dependence of $J_{e x}$ from Eq. (6) is plotted in Fig. 6, from which it is seen that the superexchange interaction diminishes with increasing Mn-O-Mn bend angle, following roughly the $\cos ^{2} \theta$ dependence of Eq. (7). For the value of $\theta=\pi / 2$, there is no hopping between the two $\mathrm{Mn}$ atoms and the $J_{e x}$ is therefore zero. For the pyrochlore compounds, the bend-angle $\theta$ being $\approx 133^{\circ}$, the magnitude of $J_{e x}$ is diminished by a factor of about 2 to a value that we estimate to be only about $\approx 4 \mathrm{meV}$, as compared to $\approx 8 \mathrm{meV}$ for the linear $\mathrm{Mn}-\mathrm{O}-\mathrm{Mn}$ bond in the perovskites. 


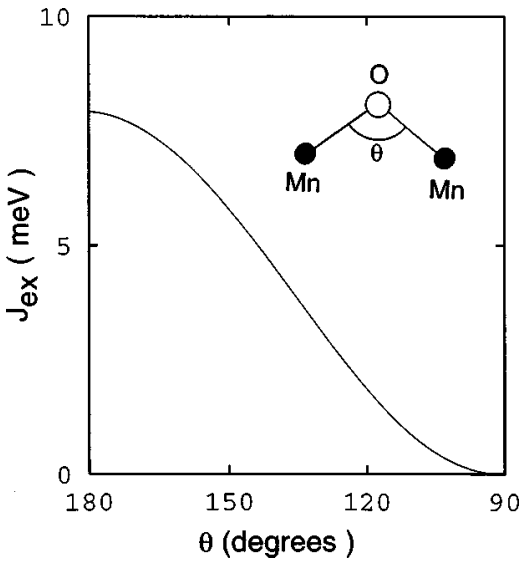

FIG. 6. Variation of the superexchange interaction between two $\mathrm{Mn}$ atoms with the Mn-O-Mn bend angle obtained from Eq. (6). Since $t / \epsilon_{d} \ll 1$, the $\cos ^{2} \theta$ dependence of Eq. (7) is followed. The parameters used are $\epsilon_{d}=6 \mathrm{eV}$ and $t=-1 \mathrm{eV}$.

\section{FERROMAGNETIC INDIRECT EXCHANGE IN THE INSULATING PYROCHLORES}

There are several pyrochlores, such as $\mathrm{Sc}_{2} \mathrm{Mn}_{2} \mathrm{O}_{7}$ and $\mathrm{Y}_{2} \mathrm{Mn}_{2} \mathrm{O}_{7}$, which are ferromagnetic insulators. However, in contrast to the metallic ferromagnets such as $\mathrm{Tl}_{2} \mathrm{Mn}_{2} \mathrm{O}_{7}$, where ferromagnetism is more robust $\left[T_{c} \sim 120 \mathrm{~K}\right.$ (Ref. 25)], the insulating pyrochlores are only weakly ferromagnetic. The weak ferromagnetism is evidenced by either a low $T_{c}$ [ $\sim 20 \mathrm{~K}$ in $\mathrm{Sc}_{2} \mathrm{Mn}_{2} \mathrm{O}_{7}$ (Ref. 26) and $\mathrm{Y}_{2} \mathrm{Mn}_{2} \mathrm{O}_{7}$ (Ref. 27)] or by the absence of a true ferromagnetic order. In fact, there are indications of a spin glass phase in these compounds, ${ }^{26,28}$ which is presumably the result of the competition between a weak ferromagnetic exchange and magnetic anisotropy. Such geometric spin frustration in a weak ferromagnetically coupled pyrochlore caused by magnetic anisotropy has been recently observed in $\mathrm{Ho}_{2} \mathrm{Ti}_{2} \mathrm{O}_{7}{ }^{29}$ Since there are no carriers present in the insulators, the Zener double exchange is absent. The question then concerns the cause of the ferromagnetic interaction.

We propose here an indirect exchange that would drive the insulating pyrochlores ferromagnetic and estimate its magnitude. This ferromagnetic interaction that is comparatively weak should indeed be present in manganites in both the pyrochlore as well as in the the perovskite structures. However, it is often masked by the stronger antiferromagnetic superexchange (e.g., in $\mathrm{LaMnO}_{3}$ ) or by the ferromagnetic DE (e.g., in Ca-doped ferromagnetic $\mathrm{La}_{1-x} \mathrm{Ca}_{x} \mathrm{MnO}_{3}$ ). It dominates in the insulating pyrochlores, since there is no $\mathrm{DE}$ and the AF superexchange is largely diminished by the bend of the Mn-O-Mn bond as discussed in the previous section. We argue that in the metallic pyrochlores such as $\mathrm{Tl}_{2} \mathrm{Mn}_{2} \mathrm{O}_{7}$, both the indirect exchange and the double exchange are present, and both being ferromagnetic interactions, the ferromagnetism is much stronger than the pyrochlore insulators, consistent with experimental observations.

The idea of the ferromagnetic indirect exchange is sketched in Fig. 7. It arises in the present model because of a small difference in the intersite Coulomb energy between the $\mathrm{Mn}$ and $\mathrm{O}$ orbitals depending on their relative spin alignment. Due to the Pauli exclusion principle, the Coulomb repulsion is slightly lower if the spins are parallel and slightly

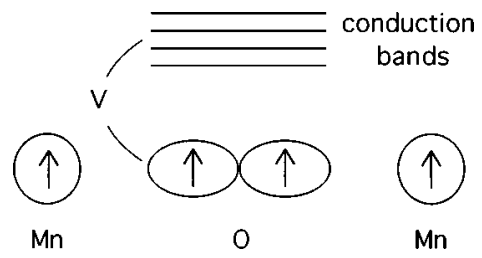

FIG. 7. Sketch of the ferromagnetic indirect exchange between Mn spins mediated via an oxygen atom. The spin polarization of the "filled-shell" oxygen orbital is produced by the coupling to the conduction-band states (see the text).

higher if they are antiparallel. The intervening oxygen atom thereby produces a net ferromagnetic Mn-Mn interaction. Now, for this mechanism to work for a filled-shell atom such as oxygen, the relevant $\mathrm{O}(2 p)$ orbitals mediating the exchange have to be coupled to other states, e.g., the conduction-band states in the crystal.

To describe this indirect exchange, we consider a FanoAnderson-type model with a single oxygen $p$ orbital (described by the creation and annihilation operators $c_{p \sigma}^{\dagger}$ and $c_{p \sigma}$ ) coupled to the conduction band (described by $c_{k \sigma}^{\dagger}$ and $\left.c_{k \sigma}\right)$ :

$$
H=\sum_{\sigma} \epsilon_{p \sigma} n_{p \sigma}+\sum_{k \sigma} \epsilon_{k} c_{k \sigma}^{\dagger} c_{k \sigma}+\sum_{k \sigma} V_{k \sigma}\left(c_{p \sigma}^{\dagger} c_{k \sigma}+\text { H.c. }\right),
$$

with the twist that the oxygen orbital energy $\epsilon_{p \sigma}$ depends on the spin orientation of the neighboring $\mathrm{Mn}$ atoms:

$$
\epsilon_{p \sigma}=\epsilon_{p}-2 \lambda\left(\vec{S}_{1} \cdot \vec{\sigma}+\vec{S}_{2} \cdot \vec{\sigma}\right) .
$$

Here $\sigma$ is the spin of the $\mathrm{O}$ electron, and $\vec{S}_{1}$ and $\vec{S}_{2}$ those of the Mn atoms, which for simplicity will be treated as classical spins of length $S=3 / 2$. The parameter $\lambda$ is the difference of the Mn-O intersite Coulomb repulsion corresponding to the parallel or antiparallel alignment of an oxygen electron spin with respect to that of a Mn electron. $\lambda$ is in fact the important parameter responsible for the FM indirect exchange.

The interaction $V_{k \sigma}$ mixes conduction-band states into the oxygen level, $\psi_{p \sigma}^{\dagger}=\alpha c_{p \sigma}^{\dagger}+\Sigma_{k} \beta_{k \sigma} c_{k \sigma}^{\dagger}$, with the corresponding energy shift of the oxygen level given by

$$
\Delta_{p \sigma}=\sum_{k} \frac{\left|V_{k \sigma}\right|^{2}}{\epsilon_{p \sigma}-\Delta_{p \sigma}-\epsilon_{k \sigma}}=-\int_{0}^{\epsilon_{\max }} \frac{V_{\sigma}^{2}(\epsilon) \rho_{\sigma}(\epsilon) d \epsilon}{\Delta_{p \sigma}-\epsilon_{p \sigma}+\epsilon},
$$

Here the energy integration runs over the conduction-band energies, from zero to $\epsilon_{\max }$, where the band bottom defines the zero of energy and $\rho_{\sigma}$ denotes the conduction-band density of states. Equation (10) may be solved iteratively from which the exchange energy may be obtained by taking the difference in energy corresponding to the $\mathrm{FM}$ and the $\mathrm{AF}$ alignment of the two Mn spins:

$$
J_{e x}=E_{\uparrow \uparrow}-E_{\downarrow \downarrow}=\sum_{\sigma} \Delta_{p \sigma}(\uparrow \downarrow)-\Delta_{p \sigma}(\uparrow \uparrow) .
$$

Here the two arrows refer to the Mn core spins and the $\sigma$ summation is over spin-up and -down states of the oxygen atom, both of which are occupied. 
Now, if we model the coupling by a constant interaction $V_{\sigma}^{2}(\epsilon) \rho_{\sigma}(\epsilon)=\mathrm{const}=V_{0}$, the energy shift is then given by

$$
\Delta_{p \sigma}=V_{0} \ln \left[\frac{\epsilon^{\max }+\Delta_{p \sigma}-\epsilon_{p \sigma}}{\Delta_{p \sigma}-\epsilon_{p \sigma}}\right] .
$$

The resulting $J_{\text {ex }}$ may be seen to scale roughly as $\lambda^{2}$ for small values of $\lambda$.

In fact, in the limit of weak interaction and a narrow conduction band, i.e., $V_{\sigma}^{2}(\epsilon) \rho_{\sigma}(\epsilon)=V^{\prime 2} \delta\left(\epsilon-\epsilon_{0}\right), V^{\prime} \ll\left(\epsilon_{0}\right.$ $\left.-\epsilon_{p}\right)$, and $\lambda \ll\left(\epsilon_{0}-\epsilon_{p}\right)$, one finds that

$$
J_{e x} \approx-\frac{V^{\prime 2} \lambda^{2}}{2\left(\epsilon_{0}-\epsilon_{p}\right)^{3}} .
$$

This mechanism therefore produces a net ferromagnetic exchange between the two Mn spins irrespective of the sign of $\lambda$, as might be expected.

There is no direct way of measuring the magnitude of $\lambda$ but we suppose that it is about $10 \%$ of the magnitude of the intersite Coulomb $U$, which is typically $\sim 0.3-0.5 \mathrm{eV}$. If as typical parameters we take $\epsilon_{p} \approx-2 \mathrm{eV}, \lambda \approx 0.05 \mathrm{eV}, V_{0}$ $\approx 1 \mathrm{eV}, \epsilon_{\text {max }} \approx 5 \mathrm{eV}$, the iterative solution of Eq. (12) results in a ferromagnetic $J_{e x} \approx 3.7 \mathrm{meV}$. Here we have incorporated an extra factor of 3 corresponding to the threefold degeneracy of the oxygen $p$ orbitals.

Even though the magnitude of the indirect exchange $J_{e x}$ depends very much on the electronic parameters and could vary from system to system, we presume that in the manganites, it is about half of the AF superexchange term for the linear Mn-O-Mn bond in accord with our above estimates. This being the case, the AF interaction dominates for the end members of the perovskite manganites $\mathrm{La}_{1-x} \mathrm{Ca}_{x} \mathrm{MnO}_{3}$. The magnitude $J_{e x} \approx 8 \mathrm{meV}$ for the linear Mn-O-Mn bond is thus reduced to about half its value by the $\mathrm{FM}$ indirect exchange, resulting in a net antiferromagnetic $J \approx 1 \mathrm{meV}\left(J_{e x}=J S^{2}\right)$, consistent with $J^{\mathrm{CaMnO}_{3}} \approx 10 \mathrm{~K}$ as estimated by Millis ${ }^{19}$ using the cubic-lattice Heisenberg result ${ }^{30} T_{N}=2.9 S(S+1) J$ and $S=3 / 2$ and the experimental $T_{N} \approx 110 \mathrm{~K}$ for $\mathrm{CaMnO}_{3} \cdot{ }^{31}$

In the insulating pyrochlores, $\mathrm{DE}$ is absent leaving the FM indirect exchange and the AF superexchange interactions to compete. Our calculations show that the latter interaction having been weakened due to the bend in the Mn-O-Mn angle, the FM indirect exchange now wins, making the system weakly ferromagnetic.

\section{SUMMARY}

In summary, we have examined the interplay of the various magnetic interactions in the manganese pyrochlores. With the three different exchange interactions, viz., the double exchange (FM), the indirect exchange (FM), and the superexchange (AF), taken into account, a consistent picture of magnetic interaction in the manganites emerges.

In the perovskite manganites $\mathrm{La}_{1-x} \mathrm{Ca}_{x} \mathrm{MnO}_{3}$, the more or less linear $\mathrm{Mn}-\mathrm{O}-\mathrm{Mn}$ bond produces a much stronger AF superexchange than the FM indirect exchange, thus turning the end members $\mathrm{LaMnO}_{3}$ and $\mathrm{CaMnO}_{3}$ antiferromagnetic. Doping with $\mathrm{Ca}$ eventually drives the system ferromagnetic due to the DE interaction mediated by the doped carriers.

In the insulating pyrochlores, the antiferromagnetic superexchange is considerably diminished owing to the large bend in the Mn-O-Mn bond, making the indirect exchange win as a result, producing a weak net ferromagnetic exchange interaction. In the metallic pyrochlores, the DE mechanism produced by the small number of Zener carriers is present as well, which makes the ferromagnetism more robust as compared to the insulating pyrochlores. This is consistent with the relatively larger value of $T_{c} \sim 120 \mathrm{~K}$ observed in the metallic pyrochlores, while in the insulating pyrochlores $T_{c}$ is either small, $\sim 20 \mathrm{~K}$, or else a clear FM phase is not observed.

The density-functional calculations for the metallic $\mathrm{Tl}_{2} \mathrm{Mn}_{2} \mathrm{O}_{7}$ compound showed interesting differences between the perovskite and the pyrochlore manganites as regards the nature of the Zener carriers. While $\mathrm{LaMnO}_{3}$ is an insulator, so that itinerant carriers must be introduced by $\mathrm{Ca}$ doping, $\mathrm{Tl}_{2} \mathrm{Mn}_{2} \mathrm{O}_{7}$, on the other hand, has a small number of itinerant Zener carriers already present, so that, even at stoichiometry, the DE mechanism is operative. These Zener carriers originate from the minority-spin $\Gamma_{1}$ band with a combination of $\mathrm{Mn} t_{2 g}, \mathrm{Tl} 6 s$, and $\mathrm{O} 2 p$ characters, but no Mn $e_{g}$ character.

Unlike the perovskites, where the Zener carrier spins are aligned parallel to the Mn core spins due to Hund's rule, the carriers in the pyrochlores prefer an antiparallel alignment. That the DE mechanism is unchanged irrespective of the spin-alignment preference of the Zener carriers with respect to the core spins is in fact already well known. ${ }^{4}$ The metallic pyrochlores can thus be considered as consisting of a small number of conduction electrons interacting via $\mathrm{DE}$ with a lattice of localized spins, which, unlike the perovskite manganites, is already weakly ferromagnetic due to indirect exchange. The conduction electrons merely strengthen the existing FM interaction via double exchange.

\section{ACKNOWLEDGMENTS}

We thank H. König for stimulating discussions. Financial support was provided by the Research Board of the University of Missouri and by the Office of Naval Research under Contract No. ONR N00014-95-1-0439.
*Present address: Department of Materials Science, Massachusetts Institute of Technology, Cambridge, MA 02139.

${ }^{1}$ Y. Shimakawa, Y. Kubo, and T. Manako, Nature (London) 379, 53 (1996).

${ }^{2}$ S.-W. Cheong, H. Y. Hwang, B. Batlogg, and L. W. Rupp, Jr., Solid State Commun. 98, 163 (1996).

${ }^{3}$ C. Zener, Phys. Rev. 82, 403 (1951).

${ }^{4}$ P. W. Anderson and H. Hasegawa, Phys. Rev. 100, 675 (1955).
${ }^{5}$ P.-G. De Gennes, Phys. Rev. 118, 141 (1960).

${ }^{6}$ M. A. Subramanian, B. H. Toby, A. P. Ramirez, W. J. Marshall, A. W. Sleight, and G. H. Kwei, Science 273, 81 (1996).

${ }^{7}$ M. A. Subramanian, G. Aravamudan, and G. V. Subba Rao, Prog. Solid State Chem. 15, 55 (1983).

${ }^{8}$ O. K. Andersen, Phys. Rev. B 12, 3060 (1975); H. L. Skriver, The LMTO Method (Springer, New York, 1983).

${ }^{9}$ D. J. Singh, Phys. Rev. B 55, 313 (1997). 
${ }^{10}$ G. H. Kwei, C. H. Booth, F. Bridges, and M. A. Subramanian, Phys. Rev. B 55, 688 (1997).

${ }^{11}$ H. D. Rosenfeld and M. A. Subramanian, J. Solid State Chem. 125, 278 (1996).

${ }^{12}$ Y. Shimakawa, Y. Kubo, T. Manako, Y. V. Sushko, D. N. Argyriou, and J. D. Jorgensen, Phys. Rev. B 55, 6399 (1997).

${ }^{13}$ See, e.g., K. Yosida, Theory of Magnetism (Springer, New York, 1996) and references therein.

${ }^{14}$ See, e.g., Y. Tomioka, A. Asamitsu, Y. Moritomo, H. Kuwahara, and Y. Tokura, Phys. Rev. Lett. 74, 5108 (1995).

${ }^{15}$ S. K. Mishra, R. Pandit, and S. Satpathy, Phys. Rev. B 56, 2316 (1997).

${ }^{16}$ S. Satpathy, Z. S. Popović, and F. R. Vukajlović, Phys. Rev. Lett. 76, 960 (1996); W. E. Pickett and D. J. Singh, Phys. Rev. B 53, 1146 (1996).

${ }^{17}$ E. J. W. Verwey, Z. Kristallogr. 91, 65 (1935); E. J. W. Verwey, P. W. Haayman, and F. C. Romeijn, J. Chem. Phys. 15, 181 (1947).

${ }^{18}$ Z. Zhang and S. Satpathy, Phys. Rev. B 44, 13319 (1991).

${ }^{19}$ A. J. Millis, Phys. Rev. B 55, 6405 (1997).

${ }^{20}$ H. König and S. Satpathy (unpublished).

${ }^{21}$ S. Chikazumi and S. H. Charap, Physics of Magnetism (Krieger, Malabar, FL, 1986); T. Nagamiya, K. Yosida, and R. Kubo,
Adv. Phys. 4, 1 (1955).

${ }^{22} \mathrm{~A}$ more detailed analysis of superexchange involving orbital degeneracy, Coulomb terms, and finite Hund's energy is presented in Ref. 20.

${ }^{23}$ W. A. Harrison, Electronic Structure and the Properties of Solids (Freeman, San Francisco, 1980).

${ }^{24}$ D. Treves, M. Eibschütz, and P. Coppens, Phys. Lett. 18, 216 (1965); G. Sawatzky, W. Geertsma, and C. Haas, J. Magn. Magn. Mater. 3, 37 (1976).

${ }^{25}$ N. P. Raju, J. E. Greedan, and M. A. Subramanian, Phys. Rev. B 49, 1086 (1994).

${ }^{26}$ J. E. Greedan, N. P. Raju, and M. A. Subramanian, Solid State Commun. 99, 399 (1996).

${ }^{27}$ J. N. Reimers, J. E. Greedan, R. K. Kremer, E. Gmelin, and M. A. Subramanian, Phys. Rev. B 43, 3387 (1991).

${ }^{28}$ J. E. Greedan, J. Avelar, and M. A. Subramanian, Solid State Commun. 82, 797 (1992).

${ }^{29}$ M. J. Harris, S. T. Bramwell, D. F. McMorrow, T. Zeiske, and K. W. Godfrey, Phys. Rev. Lett. 79, 2554 (1997).

${ }^{30}$ G. S. Rushbrooke, G. A. Baker, Jr., and P. J. Wood, in Phase Transitions and Critical Phenomena, edited by C. Domb and M. S. Green (Academic, New York, 1974).

${ }^{31}$ E. O. Wollan and W. C. Koehler, Phys. Rev. 100, 545 (1955). 